\title{
Optimized Soft Frame Design of Traditional Printing and Dyeing Process in Xiangxi Based on Pattern Mining and Edge-Driven Scene Understanding
}

Hong Xiao ( $\sim$ Xh76894005@163.com )

Changsha University of Science and Technology

\section{Research Article}

Keywords: Pattern Mining, Neural Network, Scene Understanding, Visual Interaction, Traditional Printing and Dyeing Process, Soft Computing

Posted Date: April 26th, 2021

DOl: https://doi.org/10.21203/rs.3.rs-436520/v1

License: (c) (i) This work is licensed under a Creative Commons Attribution 4.0 International License.

Read Full License 


\section{Abstract}

Traditional folk crafts are handicrafts and corresponding activities created by Chinese working people in all ages to meet their material and spiritual needs by using various material materials and handicrafts. It reflects the general characteristics of Arts and crafts, but also shows the distinctive personality of national culture. The folk printing and dyeing process of Miao Nationality in Xiangxi and Southeast Guizhou has this characteristic, which once brought a lot of convenience to people's life and became an important part of people's life at that time. However, under the impact of economic globalization, the diversity of human culture and the intangible cultural heritage of all ethnic groups are facing serious challenges. With the development of economy, the traditional manual printing and dyeing technology is gradually replaced by modern printing and dyeing technology, and its position in textile printing and dyeing is greatly reduced. However, it can not be ignored that the manual printing and dyeing process is still full of vitality, which is welcomed and loved by the public in the optimization design of fashion textiles. Traditional art carries a lot of spiritual things in its material form, so it has high aesthetic value, cultural value and profound spiritual connotation. Therefore, the optimization design of traditional printing and dyeing process framework in Xiangxi is worth studying. In this paper, using big data pattern mining algorithm and scene understanding algorithm, a framework optimization system of traditional printing and dyeing process in Xiangxi is constructed.

\section{Introduction}

For thousands of years, the Chinese nation has created a splendid material and spiritual civilization. The working people of all ethnic groups have created a large number of folk arts with their rich imagination and incomparable creativity. Among them, the traditional printing and dyeing process contains the profound cultural heritage of the Chinese nation with a long history, and also brings people unique aesthetic enjoyment. The traditional national patterns and handicrafts are replaced by modern patterns and daily necessities, and the decoration of foreign culture replaces the traditional decoration. The national folk arts and crafts are facing the crisis of subversion and deconstruction. At present, many traditional crafts have been lost and are in danger. Therefore, it is urgent to rescue and protect folk crafts $[1,2]$. As a traditional folk craft, folk printing and dyeing in Xiangxi has been listed in the national intangible cultural heritage list. It has evolved from human creative spirit and life consciousness. Xiangxi Folk printing and dyeing to meet the needs of the times, is the crystallization of labor people's hard work and wisdom [3]. Xiangxi blue calico has a history of more than 1000 years in China, which is a valuable intangible cultural wealth of our country. Xiangxi blue calico is blue and white, with unique patterns and patterns. It is widely used in clothing, daily necessities and decorations, and is welcomed and loved by the masses. In recent years, the state has paid more and more attention to the traditional crafts, and the blue calico of Western Hunan has been listed as the national intangible cultural heritage, which affirms its cultural value. However, with the development of economy and the progress of modern production technology, the development of Xiangxi blue calico is increasingly weak [4]. 
In the second half of the 19th century, with the maturity of printing and dyeing technology, the increase of population and the increase of demand, a large number of dyeing shops and large-scale specialized production and processing centers of commercial dyes came into being in Western Hunan. In addition to the hundreds of dyeing shops scattered in the counties of Western Hunan, large-scale weaving villages emerged during this period. At that time, there were tens of thousands of employees in the folk dyeing and weaving industry in Western Hunan, and the rapid development of folk dyeing and weaving industry in Western Hunan was achieved. Due to the closed geographical location and social constraints, the formation of folk printing and dyeing process in Western Hunan is a relatively slow and arduous process. For thousands of years, the ethnic minorities in Western Hunan have been exploring the printing and dyeing technology suitable for their own nation, trying to absorb nutrition from the advanced skills of the brothers in the southwest and the Han culture in the Central Plains, and finally formed a unique and very complete folk craft in Western Hunan. This traditional manual printing and dyeing process of blue calico is an extension of the Chinese humanistic spirit passed down from generation to generation in folk art. The national psychology, feelings and values expressed in folk art are very precious [5]. As the most important part of human heritage, traditional art contains the unique cultural heritage of a nation. It may be difficult to determine the economic lifeline of a country, but it can have a significant impact on the cultural richness of a nation. In order to realize the development of hand-made printing and dyeing fashionable textiles, we must have a collision with the traditional culture and realize the development in the collision of culture, so that the traditional technology has the characteristics of the times. This conforms to the aesthetic characteristics of the times and promotes the development of traditional crafts. In modern society, people begin to pursue the construction of spiritual quality after obtaining material satisfaction. Modern society advocates "individuation and liberalization". In the pursuit of fashion textiles, people need to embody their individuality, and the manual printing and dyeing process can meet people's spiritual needs. Manual printing and dyeing fashion textile design is full of humanized features, which has become a unique existence of modern textiles [8]. In order to give full play to the advantages of modern handmade printing and dyeing process, it is necessary to take the initiative to combine with art design with "fashion" characteristics, and realize the effective integration of hand printing and dyeing process, fashion patterns and textile structure $[9,10]$. If we want to study traditional crafts, we must excavate their local knowledge, understand the origin, characteristics and important cultural functions and connotations of their national crafts. In a sense, the cultural connotation of traditional crafts is the basis for the survival of crafts, so mastering the national symbols of these traditional crafts is of great significance for us to study the inheritance and dissemination of folk printing and dyeing. Through the innovative design method, while retaining the traditional manual printing and dyeing clothing process, it integrates modern innovative technology and new innovative design elements. This makes the traditional hand-made printing and dyeing clothing crafts spread in the society and play a positive role.

The ultimate goal of computer vision is to enable the computer to observe and understand the world through vision like human beings, so as to have the ability to adapt to the environment independently, which involves many sub tasks: target detection, meaningful region labeling and 3D reconstruction. As one of the basic work, image understanding has always been one of the research hotspots. As an 
extension of image understanding, the whole scene understanding of image can solve the organic integration of subtasks or image features, which is more in line with human thinking mode. Probabilistic graph model is a combination of probability theory and graph theory, which plays an important role in current image understanding. In recent decades, the graph model in the field of probability statistics and machine learning has been deeply studied, and many effective probability graph models have been proposed [11]. Probabilistic graph model provides a very effective modeling method for the overall scene understanding of images. Probability graph can not only describe the feature relationship of the image itself, but also consider the interaction between the subtasks. At present, probability graph model has been successfully applied to various image understanding models [12].

In the process of visual scene understanding and interaction, the rational use of visual information is particularly important. The semantic information extracted from it can provide accurate and intuitive scene information for virtual interactive platform. Therefore, researchers focus on accurate and efficient acquisition of multiple types of semantic information from scene images, including scene text information, object category information and scene annotation information. It is worth noting that the text information of scene plays an important role in the understanding of visual scene. Text information can be a useful supplement to the visual information in the process of scene understanding, and it can also correct the wrong recognition results. Scene annotation is the combination and extension of scene segmentation and object recognition. It needs to completely segment the object boundary in the scene and give category annotation for each pixel in the scene image. There are many differences between scene annotation and object recognition. In addition to the different action regions mentioned above, the layout information often introduced in scene labeling algorithm often plays a key role in the result of category annotation. In the process of visual scene understanding and interaction, the human-computer interaction platform will understand the semantic information obtained from the visual scene [13]. Therefore, how to use scene semantic information is a key issue in the design of human-computer interaction platform. Recently, the urgent demand for intuitive and efficient human-computer interaction has promoted the rapid development of human-computer interaction technology. The development of human-computer interaction technology is mainly reflected in the following aspects: the application of 3D interactive platform, the application of multimedia interaction mode and efficient interactive algorithm [14].

In recent years, due to the rapid development of the Internet, many industries have accumulated a large amount of data, and showing an explosive growth. In this context, data mining technology came into being. Data mining technology can not only effectively deal with a large number of historical and existing data, but also can mine valuable information from massive historical data. The traditional sequential pattern mining algorithm increases the space-time cost and sometimes causes memory overflow, which greatly reduces the performance of the traditional sequential pattern mining algorithm. In addition, the traditional sequential pattern mining algorithm mining sequence patterns quality is not high, can not meet the actual needs of customers, can not mine the most valuable information. With entering the era of big data, "massive information, lack of information" is the most common problem faced by many enterprises. How to mine the most valuable information from massive big data has become a hot 
research topic and a problem to be solved urgently $[15,16]$. Based on the above analysis, this paper constructs a framework optimization system of traditional printing and dyeing process by using scene understanding algorithm and pattern mining algorithm.

\section{Analysis Of Scene Understanding And Pattern Mining Technology 2.1. Image Understanding and Feature Engineering}

In the application field of image understanding, the modeling process of model can be divided into model feature engineering, model learning, reasoning and evaluation. Image understanding involves basic theories such as probability and statistics, graph model and machine learning. The steps of image scene understanding based on machine learning are as follows:

(1) The effective image feature engineering is constructed to find the feature engineering matching with the model.

(2) In image scene modeling and model learning, model parameters are one of the important factors that affect the performance. The best matching model parameters can be obtained by appropriate methods.

(3) To establish an effective model reasoning algorithm, this step is the output of solving practical problems. The accuracy and efficiency of the output determine the quality of the model and application scenarios.

(4) For the performance evaluation of image understanding, the evaluation of model is easy to compare and analyze, and provide reference for further improvement or research.

Feature engineering is an important concept in the field of machine learning. There is no generally accepted definition at present. It can be considered as the related work of designing feature set for machine learning application. Image understanding is an important application field of machine learning theory, and Feature Engineering naturally plays an important role in the whole image understanding. Image features refer to the attributes that can be used as signs in image scene, usually including statistical features and visual features. Image feature is the most basic processing object of image understanding, which is the basis of scene classification, image segmentation, target recognition and other tasks in image understanding.

Image understanding needs to extract a variety of data information from the image, including texture, color, spatial semantic relations, and so on, and then analyze these data information, and finally used in scene understanding. At present, the difficulty lies in how to obtain effective features suitable for specific tasks

(1) Feature extraction: there are many redundant information in the image, so it is very important to extract appropriate amount of effective image features. this 
It is the first step of image feature engineering, and the subsequent processing is based on it.

(2) Feature representation: the original extracted information can not be directly used in various machine learning models.

(3) Feature selection, learning, coding and Transformation: the features expressed properly are not necessarily all effective and need further processing. In this way, people can select, learn, encode and transform the feature data in order to meet the needs of final image understanding.

Confusion matrix is a common classification performance evaluation method in the field of pattern recognition. It describes the relationship between the real attributes in test data and the type of recognition results. The confusion matrix of multi classification (three classification as an example) problem is shown in Table 1.

Table 1

Sample confusion matrix classification

\begin{tabular}{|llll|}
\hline Real class/forecast class & A & B & C \\
\hline A & AA & AB & AC \\
\hline B & BA & BB & BC \\
\hline C & CA & CB & CC \\
\hline
\end{tabular}

Among them, $\mathrm{a}, \mathrm{B}$ and $\mathrm{C}$ are three categories, $\mathrm{AA}, \mathrm{BB}$ and $\mathrm{CC}$ represent the number of correctly identified samples, and the rest represent the number of misidentified samples. AA means the number of samples that a sample was correctly identified as a sample, $A B$ represents the number of samples that were originally a sample but were wrongly identified as sample B. other meanings are the same.

The total accuracy can be calculated by confusion matrix. The calculation formula is as follows:

$$
T A=\frac{A A+B B+C C}{A A+A B+\ldots+C C}
$$

Among them, TA represents the total accuracy, which reflects the total test accuracy of the algorithm.

Precision and recall are two quantitative indexes of classification efficiency evaluation. They can be used to evaluate the accuracy and comprehensiveness of each classification, and are also important indicators to measure the classification performance in classification system evaluation.

The precision ratio refers to the percentage of the number of relevant samples detected by classification in the total number of classified samples, which is calculated as follows: 
pre $=\left(\frac{A A}{A A+B A+C A}+\frac{B B}{B A+B B+B C}+\frac{C C}{A C+B C+C C}\right)$

Recall ratio refers to the percentage of relevant samples detected by classification in the total number of relevant samples in the system, which is calculated as follows:

$$
\text { recall }=\left(\frac{A A}{A A+A B+A C}+\frac{B B}{B A+B B+B C}+\frac{C C}{C A+C B+C C}\right)
$$

The Jaccard coefficient was originally used to compare sets. For a given class, the Jaccard coefficient can be understood as the ratio of the intersection of the real positive value and the estimated positive value set to the total positive value. Taking category $A$ as an example, the calculation formula is as follows:

$$
J C_{A}=\frac{A A}{A A+A B+A C+B A+C A}
$$

The higher the Jaccard coefficient is, the more accurate the prediction is. A stands for category, and Jaccard coefficient can be used to evaluate the classification of each category in multi classification. ROC curve is a graphical classification performance evaluation method. Figure 1 is an example of multi classification ROC curve. The horizontal axis represents the error acceptance rate (FPR), that is, the proportion of classification targets that exist but actual targets do not exist. The vertical axis represents the correct acceptance rate (TPR), that is, the proportion of classification results in which the target exists but the actual target also exists. There is usually another evaluation index when evaluating the ROC curve: the area under the curve (AUC) describes all the areas under the ROC. If the range of AUC is larger, the classification performance will be higher.

\subsection{Image Scene Annotation}

Scene annotation is one of the core tasks of visual scene understanding. It can represent the surface semantics of scene images, and is the basis of in-depth understanding of scenes. In essence, scene annotation is the combination and extension of object segmentation and object detection and recognition. It needs to completely segment the objects in the scene and give category annotation for each pixel in the scene image. In order to establish a reasonable association between image feature space and annotation semantic space, researchers introduce machine learning model to complete the task of association construction.

Scene annotation methods are classified into supervised model and unsupervised model. The supervised model needs to take the scene image with annotation information as the training data. Through the complete modeling on this basis, the scene annotation method of supervised model has a good description of the context information, object appearance and shape information in the scene. However, 
the large amount of data makes the task of collation and annotation of data sets too heavy. Many annotation databases have made more users join the tagging column by using simple and fast annotation software, thus providing researchers with real and credible large amount of data annotation data set. The unsupervised model of scene annotation method constructs a simple machine learning model to model and describe the scene data through reasonable inference and data clustering. The completeness of the results of these methods is not as good as that of the supervised model, but it requires less training data set.

The implementation process of FDSRDS algorithm is summarized as follows:

The first step: using the improved graph cutting algorithm to segment the input color image directly.

The second step: Based on the nearest neighbor graph segmentation algorithm to achieve super-pixel segmentation, the image is divided into 2 to 4 main regions.

Step 3: Based on the comparison of regional brightness based on Weber brightness, the maximum brightness region is selected as the saliency region to be reserved. Through calculation and comparison, the brightness value of the general saliency region is higher than that of other non significant regions, so the redundant non significant regions can be eliminated effectively.

Step 4: color image segmentation and binary image segmentation are realized according to the selection results.

The image cut diagram is shown in Fig. 2.

\subsection{Image Scene Understanding based on Global Context and Bayesian Topic Model}

Feature processing is both art and technology for image understanding. In the end, there is no definitive method to verify the effectiveness of feature processing in the field of machine learning. The key element of image understanding research is image pixels and their relationship. Graph model provides an appropriate expression for this. Graph model has its own rich expression vocabulary to describe the relationship between tasks and elements in image understanding. Graph model can be divided into directed graph and undirected graph. A directed graph is a graph model composed of nodes and directed edges. A node is defined as a concept or random variable, and a directed edge is defined as an edge connecting two nodes. The direction is represented by the starting node pointing to the end node. Directed graph model has its unique advantages in describing the real objective scene, so it is widely used in machine learning.

The plane graph model is the combination of probability theory and graph theory. It is the most commonly used structural model in probability and statistical analysis. It has efficient computing structure and intuitive expression. The plane diagram is composed of nodes in reasoning and expressing system and the relationship between nodes. Nodes are defined as random variables or basic concepts, 
reflecting the reason, purpose and action of the model. The relationship between nodes is represented by joint probability, which reveals the relationship of cause and effect, dependence and influence, as shown in Fig. 3.

The generation process of the plan is as follows:

(1) Sample each document class annotation.

(2) Sample topics.

(3) Each observation word was sampled.

Image understanding can be hierarchical and top-down. Firstly, given an image, secondly, extracting image feature information can form semantic feature space. Semantic feature space can be further learned to get context feature space.

\subsection{Research on Sequential Pattern Mining Algorithm}

Sequential pattern mining is a process of knowledge discovery in which frequent subsequences are found from sequential databases. That is, the process of inputting a sequence database and outputting all sequences with no less than the minimum support. Sequential pattern mining was first proposed by Agrawal and Srikant.

The basic idea of PrefixSpan algorithm is as follows: firstly, scan the sequence database, find all sequence patterns with length of 1 , and use these sequence patterns as prefixes. The sequential database is divided into several small projection databases, and then the recursive sequential pattern mining is carried out on each projection database. The effect is shown in Fig. 4. Firstly, there is a sequence database $\mathrm{s}$, which generates multiple projection databases according to the prefix division, and then recursively mines these multiple projection databases until all the frequent sequence patterns are found.

Although PrefixSpan algorithm can improve the efficiency of mining, it still has some shortcomings

First, the algorithm needs to construct a large number of projection databases, and the cost of constructing projection databases is huge. Second, the algorithm needs to scan the projection database recursively, which costs a lot of time and space, and also greatly reduces the efficiency of the algorithm.

Thirdly, the frequent sequence patterns mined by the algorithm are arranged according to the dictionary order, which can not meet the actual needs.

MapReduce programming model divides large-scale data operations to multiple sub nodes under the management of a master node, and then integrates the intermediate results of each sub node to get the final result. Simply put, MapReduce is the decomposition of tasks and the summary of results. 
The data processing process of MapReduce is mainly divided into two stages: Map stage and reduce stage, which are completed by maptask and reducetask respectively. A job usually splits the data to be processed and generates multiple maptasks according to the number of slices. Each maptask runs on a physical node to process a partition, and maps the data in the partition to an intermediate key value pair. The following figure shows how MapReduce processes data.

The following figure shows the data change model processed by MapReduce program.

The main functions of MapReduce are as follows:

(1) Client: write MapReduce code, configure jobs, and submit jobs to Hadoop cluster.

(2) Jobtracker node; this node has only one in the cluster and is responsible for initializing each job submitted by the user. A job is decomposed into multiple tasks and distributed to multiple nodes for execution. Through the heartbeat mechanism, it communicates with the node and monitors the execution status of each task. Reassign tasks that fail or slow to execute.

(3) Tasktracker node: this node has multiple nodes in the cluster. It performs tasks on the allocated data slices and communicates regularly.

(4) HDFS: the distributed file system saves the job data, including mapredcue program code and processing data), configuration information, and finally saves the job running results.

\subsection{Processing Flow of Big Data Pattern Mining}

The whole big data processing process can be summarized as the following four steps: collection, import and preprocessing, statistics and analysis, data mining.

(1) Collection: Collection refers to the collection of data from the client to the database to facilitate common query and processing. Data collection can not only use traditional relational database (mysql, Oracle), but also can use non relational database (mongodb, redis). In the process of big data collection, because of the high concurrency, a large number of databases need to be configured on the client side.

(2) Import and preprocessing: in order to analyze and mine big data, it is necessary to clean the collected big data, and then import it to the distributed storage cluster for processing. Due to the large amount of imported data, storm software is required to be used for streaming calculation of big data.

(3) Statistics and analysis: in order to meet the needs of analysis, the distributed database is used to analyze and classify the big data. Due to the large amount of data involved, the process of statistics and analysis will occupy more system resources.

(4) Data mining: it mainly implements various mining algorithms in big data, and then forecasts according to the mining results. 


\section{Construction Of Framework Optimization System For Traditional Printing And Dyeing Process}

\subsection{Innovative Design Scheme of Traditional Printing and Dyeing Handicrafts}

First of all, in the process of printing and dyeing clothing crafts, we should update the traditional concept of clothing crafts design. In the process of designing printing and dyeing patterns, we combine the design technology with traditional manual printing and dyeing technology with the characteristics of the times and modern technology concept. Integrating the aesthetic of contemporary people, so that the design of clothing crafts printing and dyeing patterns can meet the needs of modern people.

In China's traditional manual printing and dyeing process, there are various patterns and patterns, such as iris, chrysanthemum, gourd flower and cruciform, which can be realized by tie dyeing. In this regard, we can combine the modern people's aesthetic of patterns, and use traditional tie dyeing technology to diversify the patterns of clothing crafts, so as to improve the beauty of clothing crafts patterns.

Firmly green design, innovative design of traditional hand-made printing and dyeing clothing crafts. To ensure that the design of clothing crafts can meet the needs of people, reduce the harm to the environment in the production process of Arts and crafts. Innovative design of fabrics, according to the fabric composition of clothing crafts. First, the two-dimensional printing and dyeing design is carried out by hand, and then the three-dimensional structure reconstruction design is carried out. The decorative and diversified design elements are added to the fabrics of clothing crafts, so that the printed and dyed fabrics designed are close to people's life. Sticking to the naturalness of clothing handicraft materials, tie dyeing technology can be applied to modern clothing handicraft fabric printing and dyeing, which helps to develop the fabric with original ecological feeling. In the sales market of traditional hand-made printing and dyeing crafts, ecological and environmental protection is taken as the product sales positioning, so as to obtain the favor of environmentalists. The brand of environmental protection products will usher in new development for the sales of traditional handmade printing and dyeing crafts in the market.

It can optimize the use of modern computer technology, the application of computer rigid printing and dyeing, computer combined with manual way, improve and innovate the traditional manual printing and dyeing process. We can combine the paper cutting and the traditional hand printing and dyeing process of color printed calico, and break the limitations of traditional manual printing and dyeing clothing crafts. First, paper cutting is used as the basic printing and dyeing pattern plate, and then the printing and dyeing pattern is scanned and input into the computer through the scanner. According to the needs of design, designers of clothing crafts can draw up the color draft of patterns, and make them into screen version to print clothing crafts in batch.

In the optimization design of modern textiles, color fashion design is very necessary, and the commercial value of color can not be ignored. Paying attention to the color of textile design can not only meet 
people's aesthetic needs, but also convey the connotation of pattern design. China's traditional crafts include tie dyeing, batik printing, pattern dyeing and hand drawing. Through the effective combination of printing and dyeing technology and fashion art design color, the role of traditional technology is brought into play to meet the demand of modern market for fashion textiles. In the art design of textiles, attention should be paid to the practical application of color, the combination of virtual and real, and halo dyeing should be emphasized in tie dyeing process. In the pattern of printing and dyeing performance, through the bright or dark color design to highlight the strong contrast of the pattern, to meet the needs of fashion.

Modern textile printing and dyeing process is basically mature. In order to improve the fashion of textile design, we should pay attention to the design of pattern style. For example, Baroque, Rococo and Victorian style are more popular in modern times. In addition, with the rise of retro style, retro style has become one of the styles of modern textile printing and dyeing patterns. In modern society, with the alternation and circulation of time, many patterns at home and abroad are in circulation between popularity and decline. For example, the minimalist style in Germany was replaced by luxury style after it was popular for some time. Chinese style is more popular in modern China, and traditional cultural elements are mostly used in the design of traditional printing and dyeing patterns.

\subsection{Optimized Design Elements of Hand Printed Fashionable Textiles}

In the optimization design of hand printed and dyed textiles, we should not only pay attention to the integration of fashion elements, but also constantly innovate optimization design methods to improve the effect of textile printing and dyeing design. According to the market requirements, it is necessary to build a multi-disciplinary integration system, integrate printing and dyeing technology, textile product development and textile product design, realize the effective integration of system elements, and improve the effect of hand-made textile design.

In the design and development of hand-made printing and dyeing fashionable textiles, we should pay attention to the process of artistic design and realize the organic combination of artistic design and technical design. There are many kinds of design elements of hand printed fashionable textiles, which can be divided into pattern design, shading design and subsequent finishing design. Different design elements are distinguished, and different design elements are deconstructed by intuitive thinking, so as to improve the effect of construction printing and dyeing fashion textile design.

Shading design is an important part in the design of hand printed and dyed fashionable textiles, which can be regarded as the basis of the design of hand printed and dyed textiles. The quality of shading design will directly affect the overall quality of textile design. In the shade design of hand-made printing and dyeing fashionable textiles, it is necessary to understand the composition factors of shading texture, including textile fibers, woven fabrics, knitted fabrics and other textiles, because the shading design effect of different types of textiles will be quite different. 
In the optimization design of hand-made printing and dyeing fashionable textiles, we should optimize the pattern design and do a good job in the middle-level design of textile structure. Generally speaking, the pattern design mainly displays the pattern structure. The basic pattern structure includes suitable pattern, two side continuous pattern and four side continuous pattern. The pattern forms are different. In addition, there are many types of printing methods in the design of hand printed textiles, including engraving printing, digital printing and traditional manual printing and dyeing.

Due to the restriction of production technology, the image of blue calico shows a strong Abstract effect. This kind of abstraction is not an abstract which is divorced from reality and fabricated out of thin air, but a subjective representation of the objective world by folk artists. Susan Langer thinks that artistic abstraction is an abstraction different from science, mathematics and logic. It is not to abstract their common form from a certain kind of similar things by induction. What art abstracts is still a concrete thing, but it contains much more content than the real thing. Langer thinks that artistic abstraction is the abstraction of "meaningful form". Abstract thinking is one of the common and widely used forms of thinking in visual art creation. It plays a guiding role in the abstraction of natural objects in folk art, especially in blue calico. The difference between this kind of abstraction and the pure abstraction in abstract art is that it is not separated from the real world and only pursues the visual effect, but is obtained by refining and processing the objective object and retaining the main characteristics of the object. Abstract patterns on blue calico mainly include geometric patterns and auspicious symbols.

\section{Experiment And Analysis}

\subsection{Experimental Data Analysis}

In this paper, we use the 15 scenes data set, including 15 categories, a total of 4485 images. Randomly select $10 \%, 20 \%, 30 \%, 40 \%, 50 \%$ of each class as training samples, and the rest as test samples. Image features are extracted by appearance description method. Each image is represented by 12000 dimensional descriptors, and the additive kernel transform is used to obtain 36000 viterbium descriptors to represent a single image. KPCA is applied to 36000 dimensional features, and 100 principal components are extracted as input features of the model.

For the sake of preciseness, accuracy and consistency of the test data, four significant figures are reserved in the classification accuracy rate, and three significant figures or two decimal places are reserved for the time.

In the experiment, the classification accuracy under different principal components is tested, and the sampling proportion is $10 \%$. The principal components are 10, 20 and, 200, with an interval of 10 . The classifier here is SVM classifier with random super parameter optimization. The classification accuracy is shown in Fig. 7 and Fig. 8.

Page $13 / 22$ 
The data set is uploaded to HDFS and tested by the algorithm based on map reduce. The process is divided into two steps

(1) Mining sequential patterns among different categories, and then guiding the layout of different types according to the results of mining sequential patterns.

(2) Sequential pattern mining is carried out in each category, and the sequential pattern values are calculated and reordered according to the results of sequence pattern values.

The comparison of time complexity and number of species is shown in Fig. 9 and Fig. 10.

It can be seen that with the increase of support, the number of sequential patterns is reduced.

The following figure shows the confusion matrix of scene classification at the end of the model. From the figure, we can clearly see the situation of each category's wrong classification.

\section{Conclusions}

Although modern society is in the industrial society, manual printing and dyeing technology is no longer the mainstream. But in the development of printing and dyeing textiles, my mother can see that handmade printed and dyed textiles are still popular with the public. In the optimization design of modern hand printed and dyed fashionable textiles, it is necessary to integrate fashion design and technical design organically, pay attention to the fashion design of pattern, color, fashion style, etc., at the same time, pay attention to the use of innovative design methods, so as to promote the combination of fashionable aesthetics and practical use of hand printed and dyed textiles. Through the innovative design method, while retaining the traditional manual printing and dyeing clothing process, it integrates modern innovative technology and new innovative design elements. This makes the traditional hand-made printing and dyeing clothing crafts spread in the society, and play a positive impact. Xiangxi Folk printing and dyeing is the wisdom crystallization and precious heritage of Xiangxi minority people. It has gone through hardships and almost to the verge of extinction. And the inheritors of folk printing and dyeing technology have left a precious national cultural heritage for future generations in a long time. From the perspective of cultural history, folk art is indeed a kind of living fossil. It has retained the ancient life form and consciousness track of the vast majority of people for a long time. Therefore, it is particularly important to optimize the traditional printing and dyeing process framework in Xiangxi. Based on scene understanding algorithm and pattern mining technology, this paper constructs a framework optimization of traditional printing and dyeing process. The results show that the method proposed in this paper can effectively promote the development of traditional folk printing and dyeing process.

\section{Declarations}

\section{Acknowledgement}

The paper is supported by: 
The Ministry of Education Humanities and Social Science Research Youth Fund Project "Research on Revitalizing the Traditional Printing and Dyeing Technology of Xiangxi to Help Precision Poverty Alleviation" (Project Number: 19YJC760125)

\section{Conflict of interest}

The authors declare they have no conflict of interest. This article does not contain any studies with human participants or animals performed by any of the authors

\section{References}

1. Mtibaa A, 2020, October. Compute-Centric Networking At The Edge: An Autonomous Driving UseCase. In 2020 Global Information Infrastructure and Networking Symposium (GIIS) (pp. 1-6). IEEE

2. Zhu Ran (2011) Research on Inheritance and innovation of traditional folk handicraft blue calico [J]. Art and design (Theory), (06): 266-268

3. Long Xiangping. National Arts and crafts culture in Western Hunan. Liaoning Fine Arts Publishing House, 2008

4. Hossein-Nejad Z, Agahi H, Mahmoodzadeh A (2020) Image matching based on the adaptive redundant keypoint elimination method in the SIFT algorithm. Pattern Analysis and Applications, pp. $1-15$

5. Xu X (2014) Analysis on the current situation and development of blue calico technology in Tongxiang [J]. Xuezhou a edition, (6): 240

6. Yang Yuangao (2014) Characteristics and semantic interpretation of truth, goodness and beauty of Western Hunan blue calico [J]. Beauty and times, (9): 108-110

7. Zheng J, Yang P, Yang X, Chen S (2020) Truncated Low-Rank and Total p Variation Constrained Color Image Completion and its Moreau Approximation Algorithm. IEEE Trans Image Process 29:78617874

8. Wang Yihan W Liping. Discussion on the development of textile printing and dyeing enterprises under the new economic situation [J]. Dyeing and finishing technology, 2015 (2): 32-35

9. Zhao Yue (2014) Creative design of traditional handmade printing and dyeing clothing products [J]. Industry and Technology Forum, (12): 217-218

10. Chai Songhua. Research and development of tourism costumes of ethnic minorities in Xiangxi: Research on printing and dyeing patterns. Master's thesis of Xi'an University of engineering, 2010

11. Gao, Jun (2009) Xie Zhao. Theory and method of image understanding [M]. Science Press, Beijing, pp 85-90

12. Felzenszwalb PF, Girshick RB, McAllester D et al (2010) Object detection with discriminatively trained part-based models[J]. IEEE Trans Pattern Anal Mach Intell 32(9):1627-1645

13. Li LJ, Socher R, Li FF. Towards total scene understanding: Classification, annotation and segmentation in an automatic framework[C]. Proceedings of the IEEE Conference on Computer 
Vision and Pattern Recognition, CVPR 2009, Miami, FL, United states, 2009, 2036-2043

14. Hernandez-Matas C, Zabulis X, Argyros AA (2020) REMPE: Registration of retinal images through eye modelling and pose estimation. IEEE journal of biomedical health informatics 24(12):3362-3373

15. Wang Hu (2009) Ding Shifei. Research and development of sequential pattern mining [J]. Computer science 36(12):14-17

16. Wang Y, Zhang J, Cavichini M, Bartsch DUG, Freeman WR, Nguyen TQ, An C (2021) Robust ContentAdaptive Global Registration for Multimodal Retinal Images Using Weakly Supervised Deep-Learning Framework. IEEE Trans Image Process 30:3167-3178

\section{Figures}

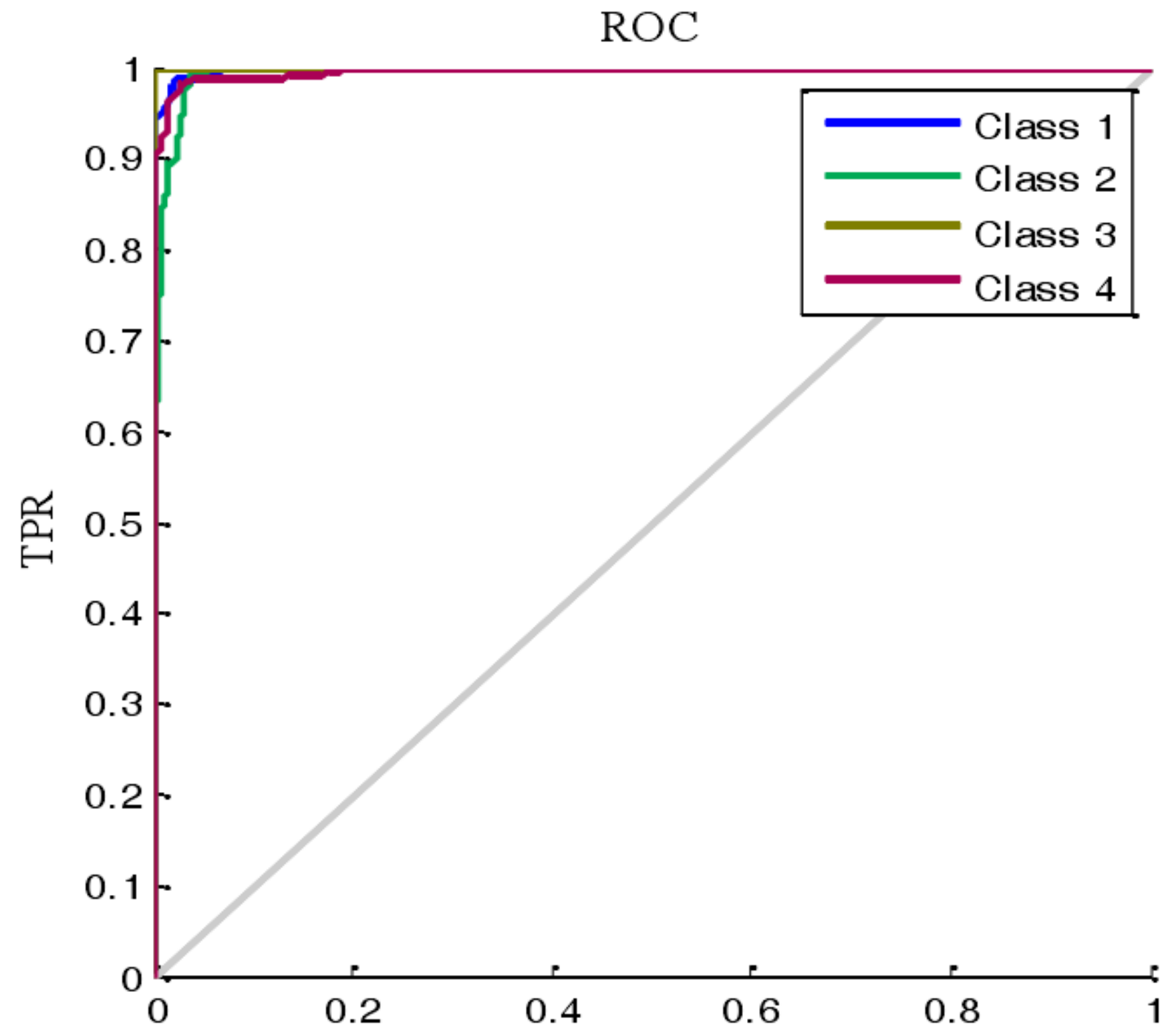

Figure 1 
ROC curve

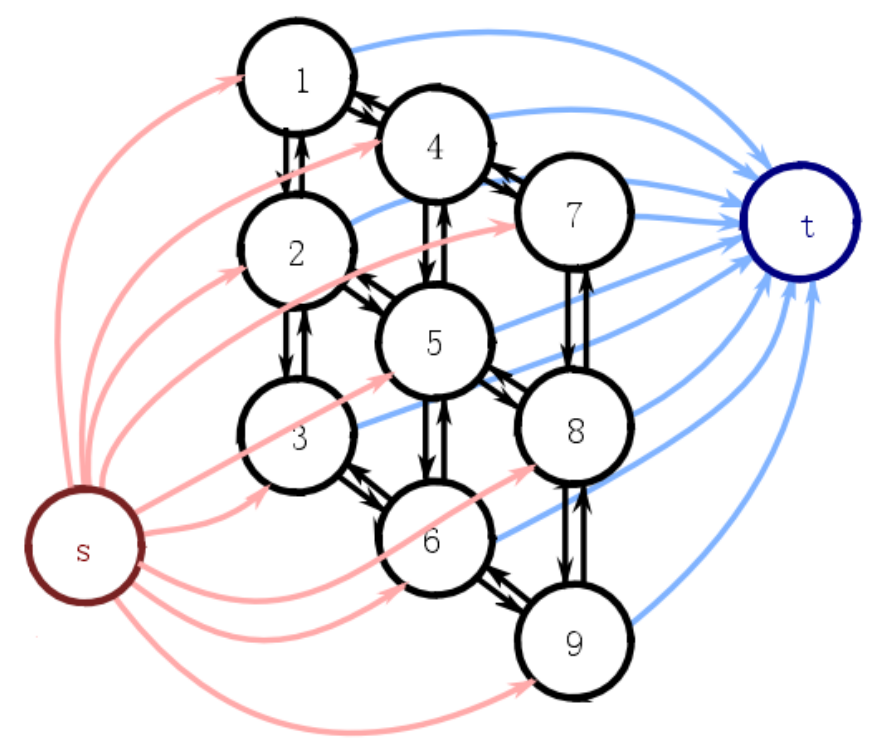

(a)

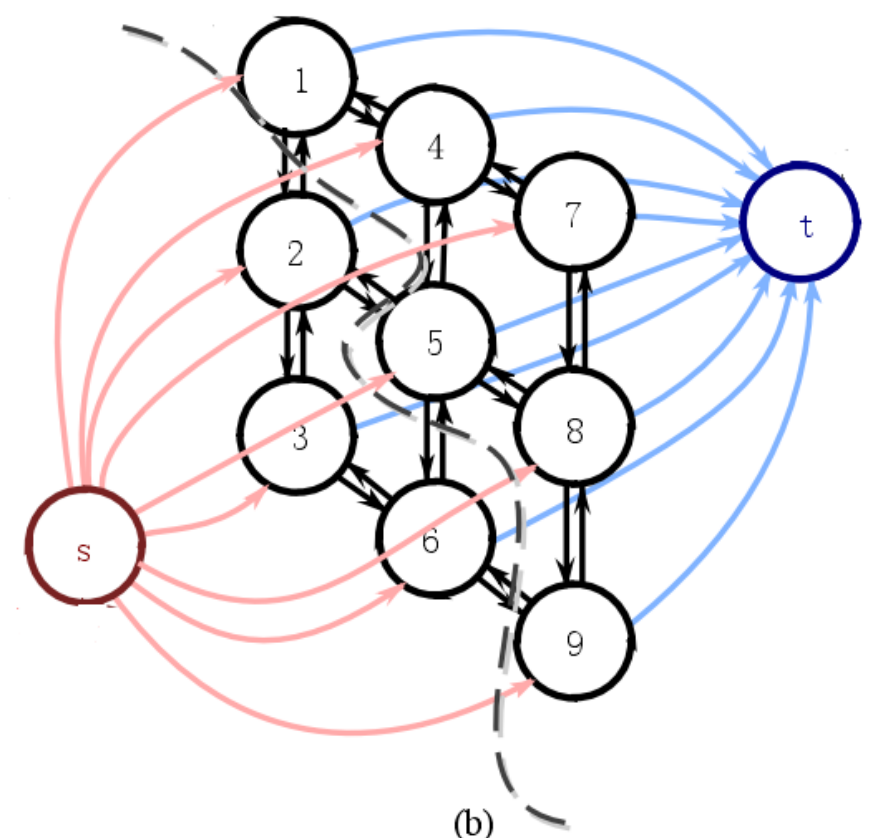

(b) -

Figure 2

Image cut

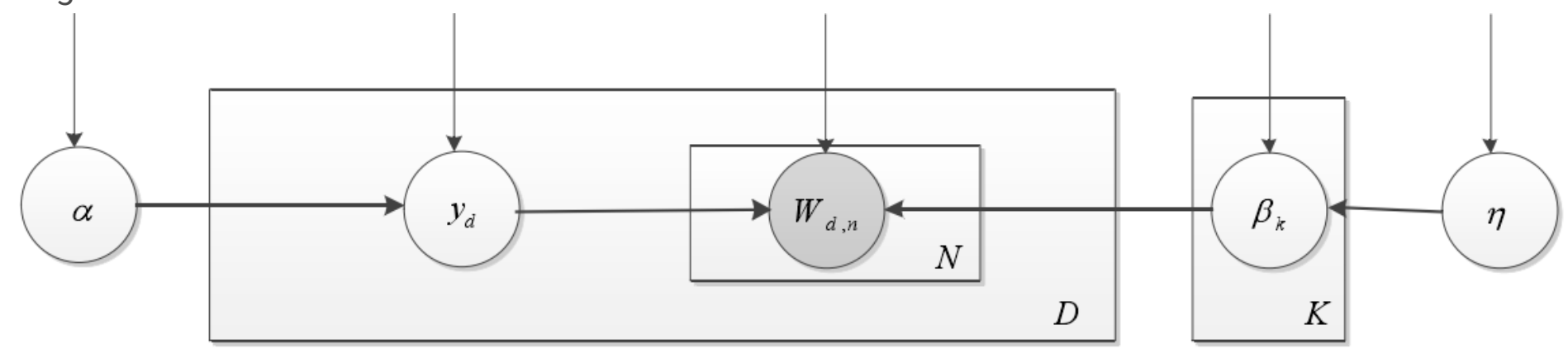

Figure 3

Plan model 


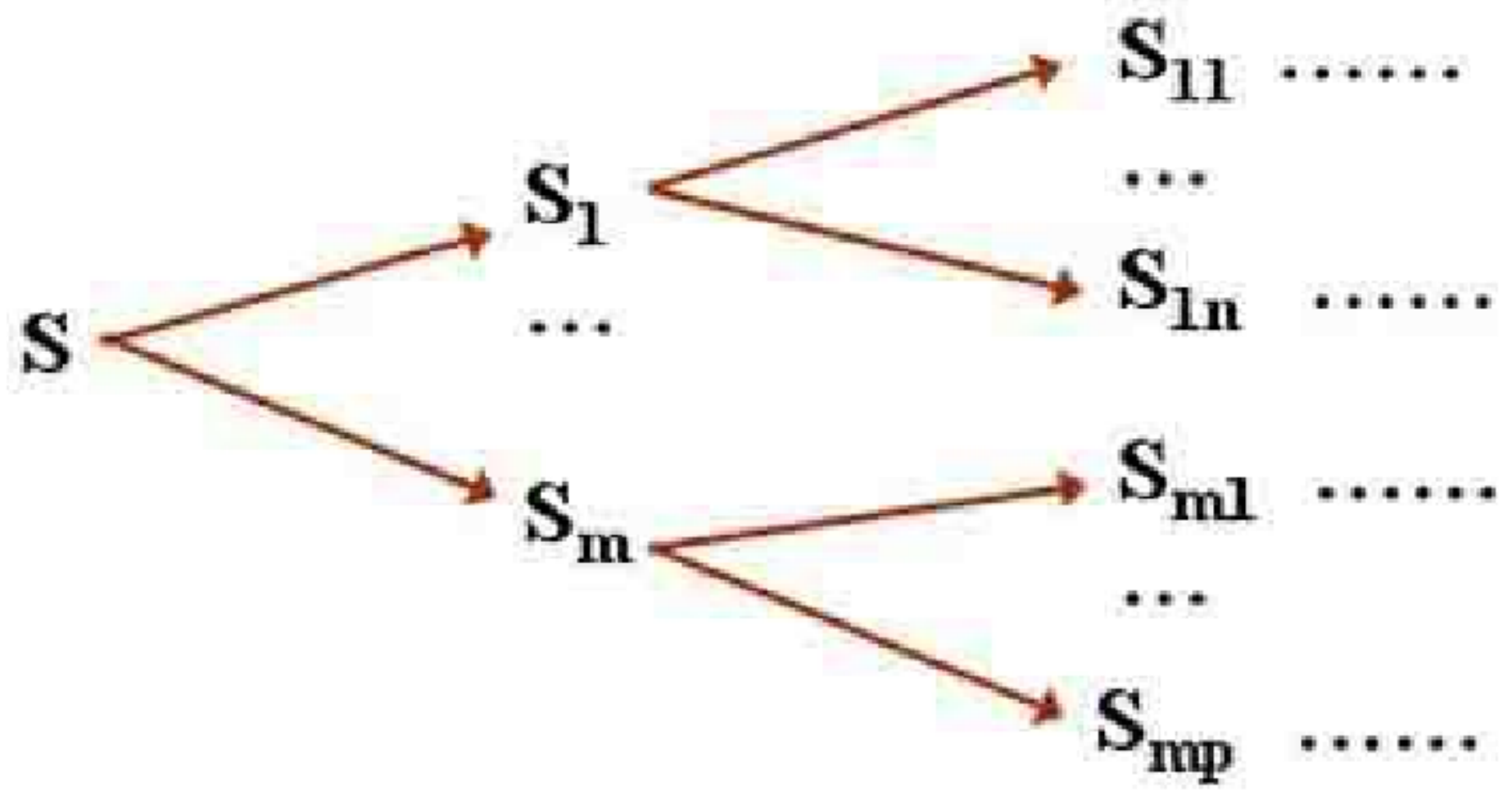

Figure 4

PrefixSpan algorithm

Input

Map

Reduce

Output

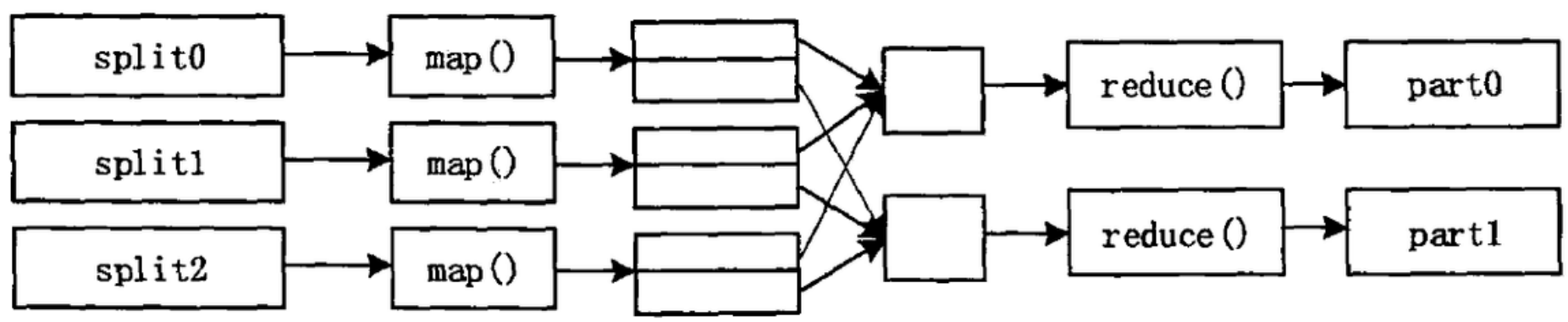

Figure 5

MapReduce data processing process

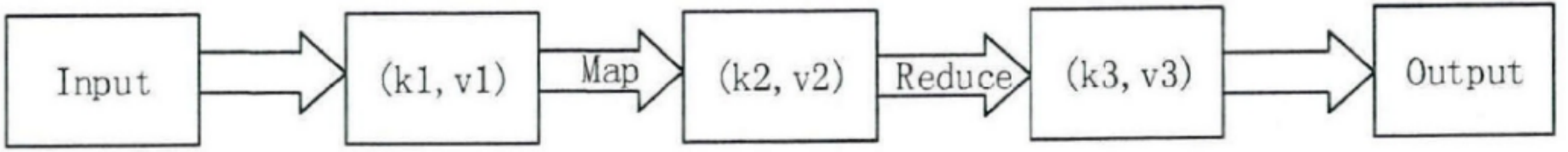

Figure 6

The basic model of MapReduce program data change 


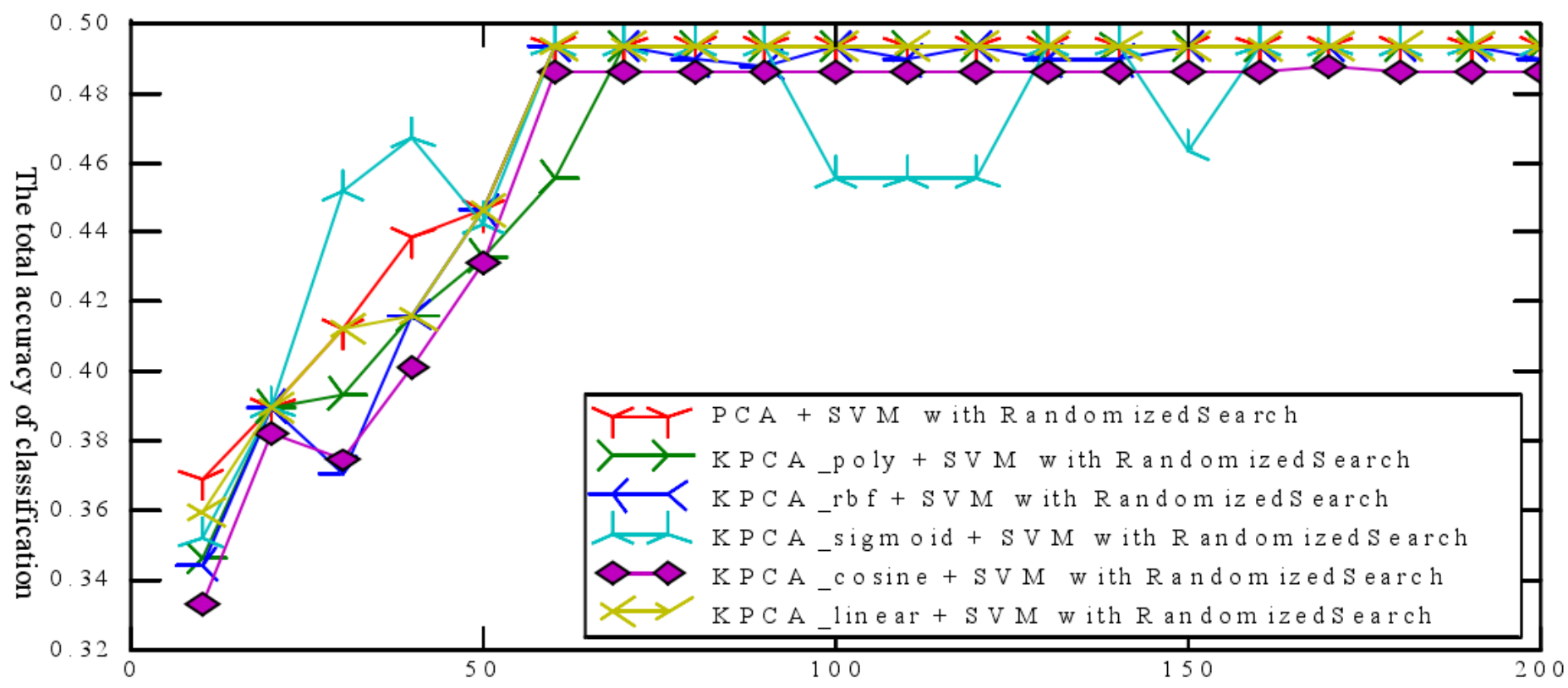

The PCA and KPCA components extracted on Msrc-v2 data set with $10 \%$ sampling

\section{Figure 7}

Comparison of classification accuracy under different number of principal components at $10 \%$ sampling ratio

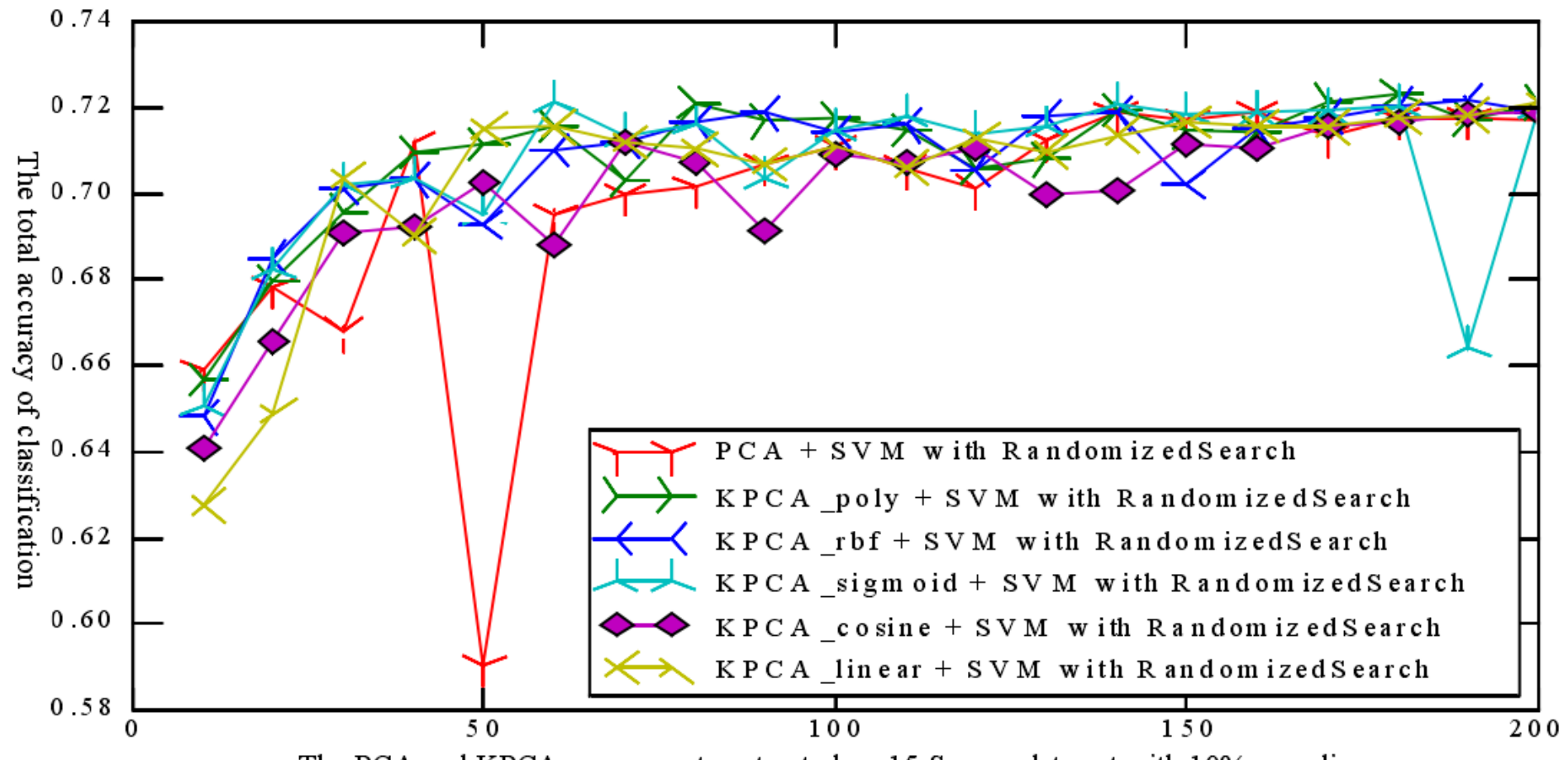

The PCA and KPCA components extracted on 15 -Scenes data set with $10 \%$ sampling

\section{Figure 8}

Comparison of classification accuracy under different number of principal components at $10 \%$ sampling ratio 


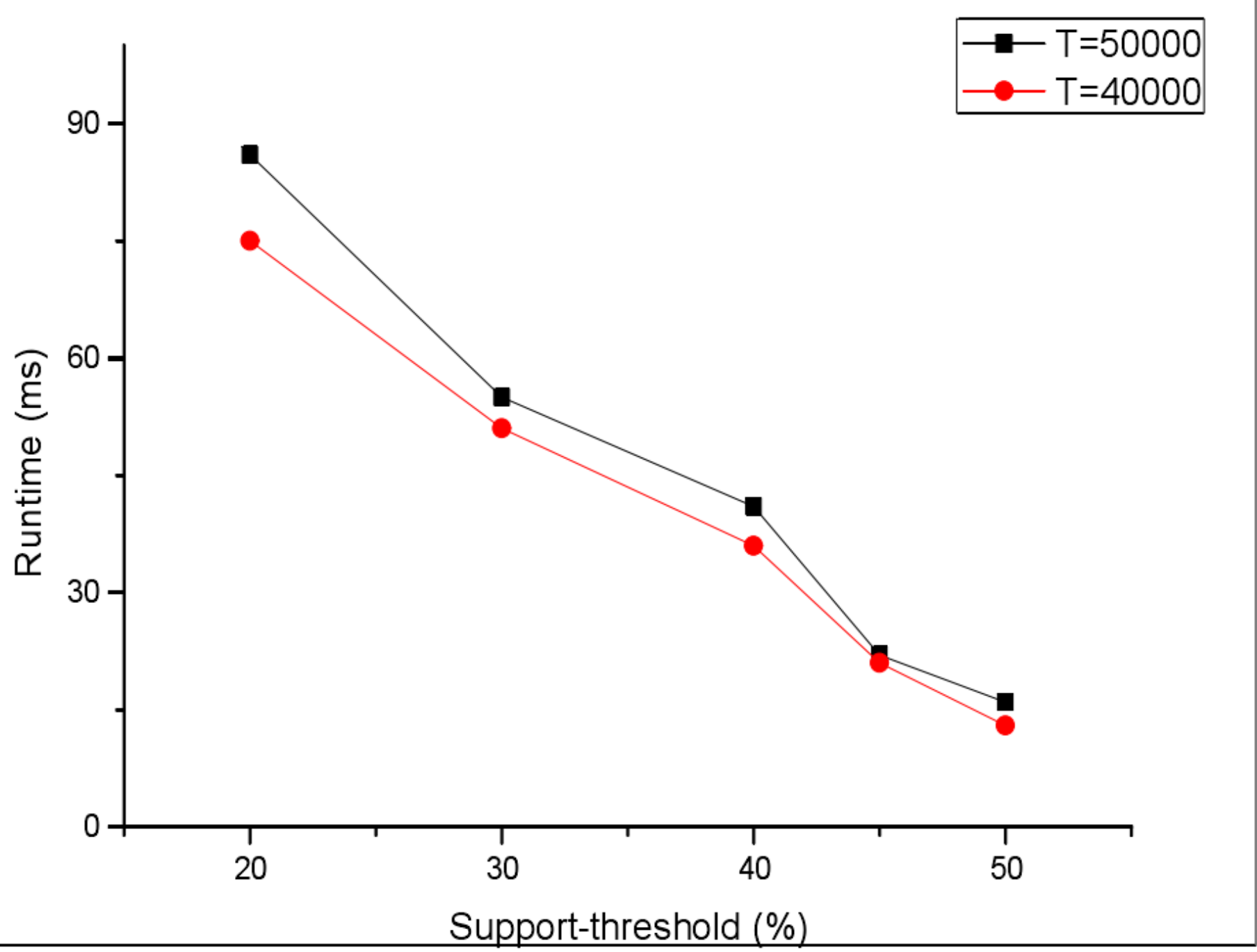

Figure 9

Comparison of mining run time between species 


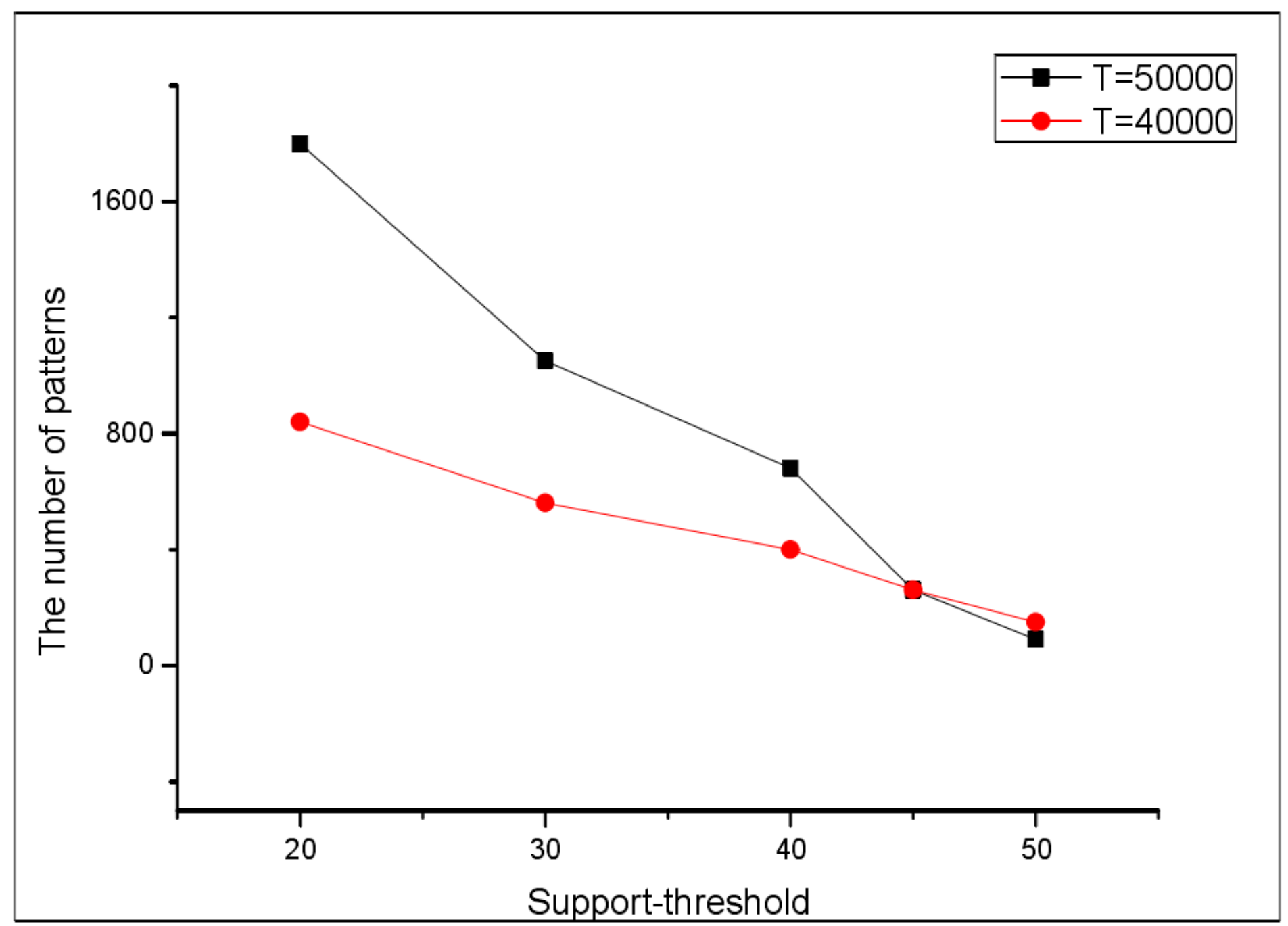

Figure 10

Comparison of the number of frequent itemsets mined among species 


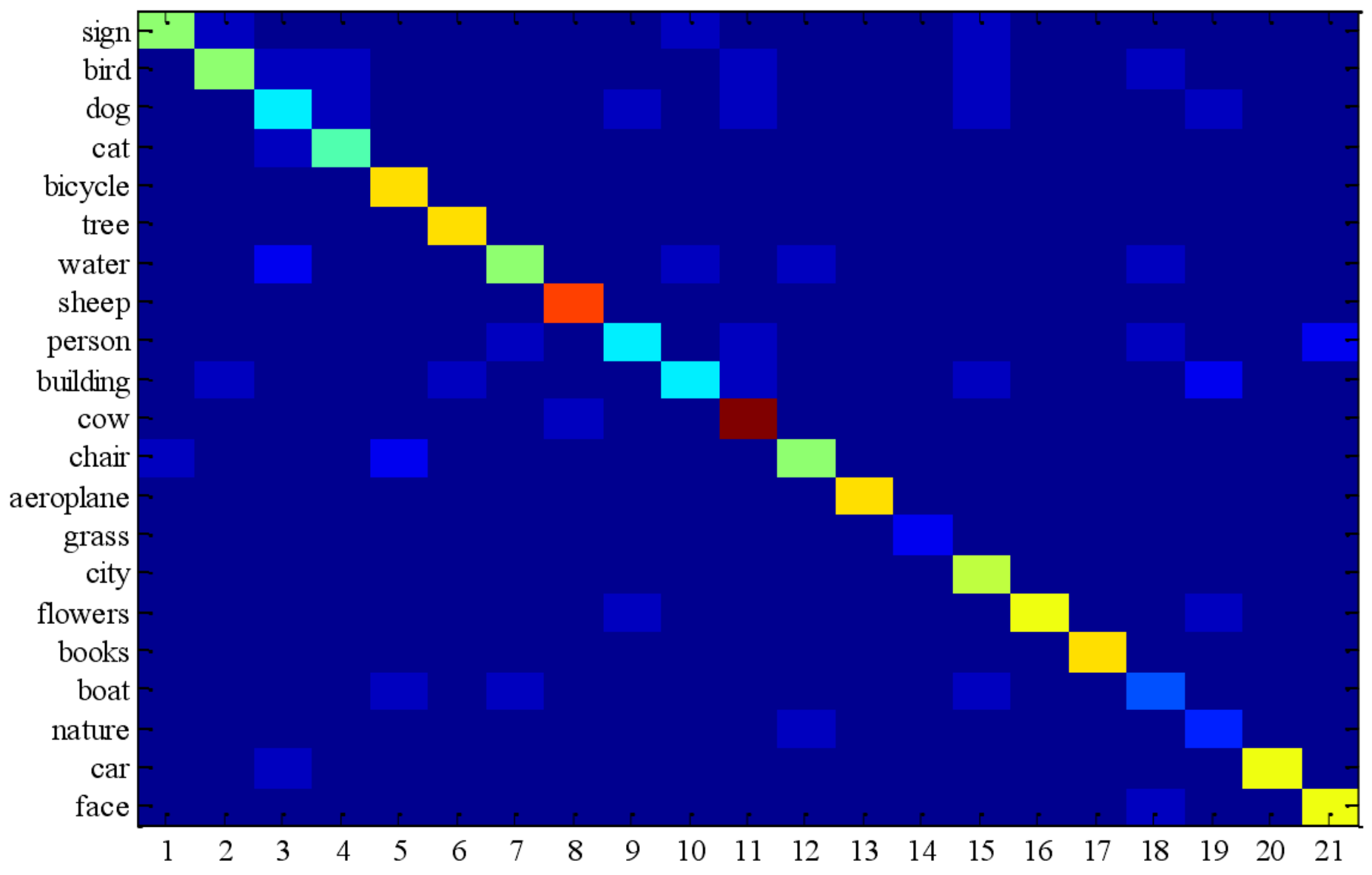

Figure 11

Classification confusion matrix of global scene model 